\title{
FINITE GROUPS WITH A MINIMAL FRATTINI SUBGROUP PROPERTY
}

\author{
JOSEPH KIRTLAND \\ Department of Mathematics, Marist College, Poughkeepsie, NY 12601 \\ e-mail: joe.kirtland@marist.edu
}

(Received 21 May, 2001; accepted 30 May, 2002)

\begin{abstract}
Finite groups in which the Frattini subgroup of each proper normal subgroup is trivial, while the group itself has a nontrivial Frattini subgroup, are investigated. A direct result of this study leads to a classification of finite groups in which the Frattini subgroup of each proper subgroup is trivial, while the group itself has a nontrivial Frattini subgroup.
\end{abstract}

2000 Mathematics Subject Classification. Primary 20D25, Secondary 20 E34.

1. Introduction. A group $G$ is called an elementary group if the Frattini subgroup of each subgroup of $G$ is trivial. Elementary groups, which were introduced and studied by Bechtell in [1], have elementary abelian Sylow subgroups and each normal subgroup is complemented. More precisely, he showed that for a finite group the three conditions are equivalent. Bechtell, also in [1], generalized elementary groups through the study of E-groups, which have the property that the Frattini subgroup of each subgroup is contained in the Frattini subgroup of the group. Tani Corsi, in [6] and [7], extended these approaches by imposing a minimal condition on the Frattini subgroup. A group $G$ is called a proper $\mathcal{F}$-group if the Frattini subgroup of $G$ is nontrivial, but the Frattini subgroup of each proper subnormal subgroup is trivial. Her investigation resulted in necessary and sufficient conditions for when a group with a nilpotent derived subgroup is a proper $\mathcal{F}$-group.

This motivates the study of finite groups which have a minimal Frattini subgroup condition and satisfy certain Frattini subgroup containment properties. The first natural approach is to study finite groups in which the Frattini subgroup of each proper subgroup is trivial, while the group itself has a nontrivial Frattini subgroup. This class of groups is classified in Corollary 3.3. The next step, motivated by Corollary 3.3 and the fact that the Frattini subgroup of a normal subgroup is always contained in the Frattini subgroup of the group, is an investigation of finite groups in which the Frattini subgroup of each proper normal subgroup is trivial, while the group itself has a nontrivial Frattini subgroup.

The notation used is standard. For a group $G, \Phi(G)$ denotes its Frattini subgroup, $F(G)$ denotes its Fitting subgroup, $Z(G)$ denotes its center, and $G^{\prime}$ its derived subgroup. A normal subgroup $N$ of a group $G$ has a complement in $G$ if there is a subgroup $H$ of $G$ such that $G=N H$ and $N \cap H=\{1\}$. In this case, $G$ is said to split over $N$ and is denoted $G=[N] H$. All groups considered are finite.

To make the terminology less cumbersome, we make the following definitions. 
Definition 1.1. A group $G$ is a minimal non-elementary group if $\Phi(G) \neq\{1\}$, but $\Phi(H)=\{1\}$ for all proper subgroups $H$ of $G$.

Definition 1.2. A group $G$ is proper $\mathcal{N} \mathcal{F}$-group if $\Phi(G) \neq\{1\}$, but $\Phi(N)=\{1\}$ for all proper normal subgroups $N$ of $G$.

2. Preliminary results. In this section, some general results concerning proper $\mathcal{N} \mathcal{F}$-groups are given.

LemMa 2.1. Let $G$ be a proper $\mathcal{N} \mathcal{F}$-group. Then

(i) $G$ is direct product indecomposable;

(ii) all nilpotent proper normal subgroups of $G$ are elementary abelian. In particular, $\Phi(G)$ is elementary abelian;

(iii) the Fitting subgroup $F(G)$ exists nontrivially in $G$. Furthermore, if $F(G) \neq G$, then $F(G)$ is elementary abelian.

Proof. To prove (i), suppose $G=N_{1} \times N_{2}$, where $N_{1} \neq\{1\}$ and $N_{2} \neq\{1\}$. Since $N_{1}$ and $N_{2}$ are both proper normal subgroups in $G, \Phi\left(N_{1}\right)=\Phi\left(N_{2}\right)=\{1\}$. This implies that $\Phi(G)=\{1\}$, a contradiction.

To prove (ii), let $N$ be a nilpotent proper normal subgroup of $G$. Since $\Phi(N)=\{1\}$, $N$ must be elementary abelian.

The first part of statement (iii) follows from the fact that $\Phi(G)$ is nilpotent and exists nontrivially in $G$. If $F(G) \neq G$, the second part follows from (ii).

THEOREM 2.2. Let $G$ be a nonabelian proper $\mathcal{N} \mathcal{F}$-group. Then $Z(G) \leq \Phi(G)$.

Proof. By Lemma 2.1(ii), $Z(G)$ is elementary abelian. Assume there is an element $x \in Z(G) \backslash \Phi(G)$. Then $\langle x\rangle \cap \Phi(G)=\{1\}$, where $\langle x\rangle$ is an abelian normal subgroup of $G$. Thus, by 5.4.10 of [2], $G=[\langle x\rangle] H=\langle x\rangle \times H$ for some $H<G$. This is a contradiction by Lemma $2.1(i)$.

Therefore $Z(G) \leq \Phi(G)$.

3. Main results. In this section, results are obtained concerning the structure of certain classes of proper $\mathcal{N} \mathcal{F}$-groups. The classification of minimal non-elementary groups is presented as a corollary to Theorem 3.2.

Proposition 3.1. A minimal non-elementary group is a p-group, for some prime p.

Proof. Let $G$ be a minimal non-elementary group. Suppose that $G$ has two or more Sylow subgroups. Then each Sylow subgroup is a proper subgroup of $G$ and must have a trivial Frattini subgroup. By Theorem 2.3 in [1], this implies that $\Phi(G)=\{1\}$, a contradiction. Thus $G$ is a $p$-group.

THEOREM 3.2. A nilpotent group $G$ is a proper $\mathcal{N} \mathcal{F}$-group if and only if $G$ is cyclic of order $p^{2}, p$ any prime, or a nonabelian elementary $p$-group of order $p^{3}, p$ an odd prime.

Proof. Suppose $G$ is a nilpotent proper $\mathcal{N} \mathcal{F}$-group. Then $G$ is a $p$-group by Lemma 2.1(i).

If $G$ is abelian, then again by Lemma 2.1(i), $\mathrm{G}$ is cyclic. Thus $G=\langle a\rangle$, where $|a|=p^{\alpha}$, and by Lemma 2.1(ii), $\Phi(G)$ is elementary abelian. Since $\Phi(G) \neq\{1\}, \alpha=2$ and $G$ is cyclic of order $p^{2}$. 
If $G$ is not abelian, then $|G|=p^{\alpha}$, with $\alpha \geq 3$, and $Z(G) \leq \Phi(G)$ by Theorem 2.2. For any element $y \in G$, the subgroup $\Phi(G)\langle y\rangle$ is proper in $G$ since $G$ is not abelian, and normal in $G$ since $G^{\prime} \leq \Phi(G)$. Thus $\Phi(G)\langle y\rangle$ is elementary abelian by Lemma 2.1(ii). Therefore $G$ is of exponent $p$, with $p$ odd, and $\Phi(G)$ is contained in $Z(G)$. Hence $G^{\prime}=\Phi(G)=Z(G)$.

Now let $v$ and $w$ be two non-commuting elements of $G$. From $\Phi(G)\langle v, w\rangle$ nonabelian, it follows that $G=\Phi(G)\langle v, w\rangle=\langle v, w\rangle$; therefore $\left|G^{\prime}\right|=p$ and $|G|=p^{3}$.

The converse is clear.

COROllary 3.3. A group $G$ is a minimal non-elementary group if and only if $G$ is cyclic of order $p^{2}, p$ any prime, or a nonabelian elementary $p$-group of order $p^{3}, p$ an odd prime.

Proof. By Proposition 3.1 the result follows immediately from Theorem 3.2.

The case for non-nilpotent supersolvable proper $\mathcal{N} \mathcal{F}$-groups is much more involved. The investigation here is limited to certain types of non-nilpotent supersolvable groups whose order is divisible by two distinct primes. First a preliminary lemma is given.

LEMMA 3.4. Let $G$ be a non-nilpotent supersolvable proper $\mathcal{N} \mathcal{F}$-group, let $p$ be the largest prime dividing the order of $G$ and let $S_{p}$ be the Sylow p-subgroup of $G$. Then

(i) $S_{p}$ is elementary abelian;

(ii) $G=\left[S_{p}\right] H$, where $H$ is a Hall $p^{\prime}$-subgroup of $G$ with $\Phi(G)<H$;

(iii) $G$ is metabelian;

(iv) $S_{p}<F(G)$.

Proof. Since $S_{p}$ is a proper normal subgroup of $G$, it is elementary abelian by Lemma 2.1(ii).

To prove (ii), suppose that $p$ divides $|\Phi(G)|$. Then $\Phi(G) \cap S_{p}=N \neq\{1\}$, where $|N|=p^{\alpha}, \alpha \geq 1$. By Lemma 2.1(ii), $\Phi(G)$, and thus $N$, are elementary abelian. Since $S_{p}$ is elementary abelian, $S_{p}$ splits over $N$. By a result due to Gaschütz (4.2.4 in [2]), $G$ must also split over $N$. This is a contradiction as $N \leq \Phi(G)$. Thus $p$ does not divide $|\Phi(G)|$ and $\Phi(G)$ is a $p^{\prime}$-subgroup of $G$. Since $G$ is solvable, $\Phi(G)$ is contained in a Hall $p^{\prime}$-subgroup $H$ of $G$. As a result, $G=\left[S_{p}\right] H$, where $\Phi(G)<H$.

To prove (iii), notice that $G^{\prime}$ is nilpotent since $G$ is supersolvable. Consequently, by Lemma 2.1(ii), $G^{\prime}$ is elementary abelian and $G$ is metabelian.

Finally (iv) follows immediately from (ii).

While not within the same context of this paper, it should be noted here that metabelian groups with abelian Fitting subgroups have been studied by Steffen in [4] and [5].

THEOREM 3.5. Let $G$ be a non-nilpotent supersolvable proper $\mathcal{N} \mathcal{F}$-group of order $p^{\alpha} q^{\beta}, p$ and $q$ primes with $p>q$, and having abelian Sylow $q$-subgroups. Let $S_{p}$ be the Sylow p-subgroup of $G$ and $S_{q}$ be a Sylow q-subgoup of $G$. Then $G=\left[\left\langle a_{1}\right\rangle \times \cdots \times\left\langle a_{\alpha}\right\rangle\right] S_{q}$, where $\left|a_{i}\right|=p$ and $\left\langle a_{i}\right\rangle$ normal in $G$ for any $i, \Phi(G)<S_{q}$, and $G^{\prime}=S_{p}$.

Proof. By Lemma 3.4, $\Phi(G)<S_{q}$ and $S_{p}$ is elementary abelian, with $S_{p}=$ $\left\langle a_{1}\right\rangle \times \cdots \times\left\langle a_{\alpha}\right\rangle$. By 5.4.10 of [2], each $\left\langle a_{i}\right\rangle$ is normal in $G$. 
Obviously $G^{\prime} \leq S_{p}$. Since $S_{q}$ is a $p^{\prime}$-group acting on $S_{p}, S_{p}=C_{S_{p}}\left(S_{q}\right) \times\left[S_{p}, S_{q}\right]$ by 5.3.5 of [3], where $\left[S_{p}, S_{q}\right]=G^{\prime}$ and $C_{S_{p}}\left(S_{q}\right)=Z(G) \cap S_{p}$ (see also 8.3.6 of [2]). Since $Z(G) \leq \Phi(G)$, by Theorem 2.2, $Z(G) \cap S_{p}=\{1\}$ and $G^{\prime}=S_{p}$.

COROLlaRY 3.6. Let $G$ be a non-nilpotent supersolvable proper $\mathcal{N} \mathcal{F}$-group of order $p^{\alpha} q^{\beta}, p$ and $q$ primes with $p>q$, and having cyclic Sylow $q$-subgroups. Let $S_{p}$ be the Sylow p-subgroup of $G$ and $S_{q}=\langle x\rangle$ be a Sylow q-subgroup of $G$. Then $G=$ $\left[\left\langle a_{1}\right\rangle \times \cdots \times\left\langle a_{\alpha}\right\rangle\right]\langle x\rangle$, where $\beta=2,\left|a_{i}\right|=p$ and $\left\langle a_{i}\right\rangle$ normal in $G$ for any $i, \Phi(G)=$ $Z(G)=\left\langle x^{q}\right\rangle$, and $G^{\prime}=S_{p}$.

Proof. By Theorem 3.5, $\Phi(G)<\langle x\rangle, S_{p}$ is elementary abelian where $S_{p}=$ $\left\langle a_{1}\right\rangle \times \cdots \times\left\langle a_{\alpha}\right\rangle$ with each $\left\langle a_{i}\right\rangle$ is normal in $G$, and $G^{\prime}=S_{p}$. Moreover $\Phi(G) \neq\{1\}$ implies $\beta \geq 2$ and $x^{q^{\beta-1}} \in \Phi(G)$. Thus $S_{p}\left\langle x^{q^{\beta-1}}\right\rangle=S_{p} \times\left\langle x^{q^{\beta-1}}\right\rangle \leq F(G)$.

Suppose that $\beta \geq 3$ and consider the proper normal subgroup $N=\left[S_{p}\right]\left\langle x^{q}\right\rangle$ of $G$. Since $x^{q^{\beta-1}}$ is in the center of $N, x^{q^{\beta-1}}$ is in each maximal subgroup of $N$. This contradiction implies that $\beta=2$. Consequently $\Phi(G)=Z(G)=\langle x\rangle$.

ACKNOWLEDGEMENT. The author is very grateful for the numerous comments and suggestions made by the referee.

\section{REFERENCES}

1. H. Bechtell, Elementary groups, Trans. Amer. Math. Soc. 114 (1965), 355-362.

2. H. Bechtell, The theory of groups (Addison-Wesley, 1971).

3. D. Gorenstein, Finite groups (Harper and Row, 1968).

4. G. Steffen, Zur Struktur endlicher metabelscher Gruppen mit abelscher Fitting-gruppe. I., Beiträge Algebra Geom. 15 (1983), 67-82.

5. G. Steffen, Zur Struktur endlicher metabelscher Gruppen mit abelscher Fitting-gruppe. II., Beiträge Algebra Geom. 16 (1983), 69-78.

6. G. Tani Corsi, Sui Gruppi Finiti in cui ogni Sottogruppo Subnormale Proprio è a Sottogruppo di Frattini Identico, Matematiche (Catania) 21 (1966), 131-134.

7. G. Tani Corsi, Una Caratterizzazione dei Gruppi Finiti a Derivato Nilpotente in cui ogni Sottogruppo Subnormale Proprio ha Sottogruppo di Frattini Identico, Matematiche (Catania) 22 (1967), 19-23. 